How to reference this article Serena, E. (2018). Modelli di descrizione grammaticale nei manuali di italiano per apprendenti di madrelingua tedesca. Italica Wratislaviensia, 9(1), 247-269.

DOI: http://dx.doi.org/10.15804/IW.2018.09.13

Enrico Serena

Ruhr-Universität Bochum

\title{
MODELLI DI DESCRIZIONE GRAMMATICALE NEI MANUALI DI ITALIANO PERAPPRENDENTIDIMADRELINGUATEDESCA
}

\author{
MODELS OF GRAMMATICAL DESCRIPTION \\ IN ITALIAN TEXTBOOKS \\ FOR GERMAN-SPEAKING LEARNERS
}

\begin{abstract}
This paper investigates the conceptual and terminological framework employed in the grammatical sections of Italian textbooks for German-speaking adult learners. The paper especially aims to establish what relationship these sections have with the framework of traditional grammar (TG) and models of grammatical description in modern linguistics (ML). The analysis takes the following variables into account: 1) treatment of parts of speech, paying particular attention to manners of presentation and criteria of definition; 2) presence or absence of explicit or implicit references to the concept of "phrase"; 3) treatment of the syntactic functions of subject, direct and indirect objects, and complements; and 4) employed terminology. The analysis leads to several conclusions. First, the most remarkable differences in the treatment of parts of speech from textbook to textbook concern articles and transversal classes such as demonstratives, indefinites, and possessives. Additionally, when parts of speech are explicitly defined, the definitions do not follow homogeneous criteria. Furthermore, references to the concept of "phrase", fundamental in ML and unknown or ignored in TG, are only implicit. Moreover, though the functions of subject, object, and complements are mentioned often, they are never explicitly defined. Finally, terminological choices are somewhat eclectic, as they include terms from international metalinguistic terminology as well as technical vocabulary from the German and Italian school traditions.
\end{abstract}

Keywords: grammar, Italian textbooks, parts of speech, syntactic functions, metalinguistic terminology 


\section{INTRODUZIONE}

T manuali per l'insegnamento/apprendimento delle lingue straniere si 1 compongono tradizionalmente di diverse parti: oltre ai capitoli per il lavoro in classe, essi contengono di norma anche un numero variabile di sezioni tematiche. Tra queste, occupano sicuramente un posto di rilievo le sezioni grammaticali (SG), che rappresentano un prezioso strumento di consultazione sia per l'apprendente che per il/la docente. Il/la primo/a vi può infatti ricorrere per approfondire gli argomenti trattati in classe e per aiutarsi durante lo svolgimento degli esercizi. Il/la secondo/a può d'altra parte servirsene durante la preparazione della lezione sia per trovare spiegazioni metalinguistiche ed esempi da proporre in classe sia come prima fonte per chiarirsi egli/ella stesso/a dubbi di varia natura. Le SG, talvolta anche contenute in un fascicolo esterno al libro dello studente, possono ricalcare nella loro struttura la partizione del manuale in capitoli e presentare quindi gli argomenti grammaticali nell'ordine della loro occorrenza nelle unità per il lavoro in classe oppure possono avere il carattere di grammatica sistematica e quindi essere suddivise in paragrafi tematici ${ }^{1}$ come le grammatiche non legate a manuali. Per la loro funzione di supporto dei processi di insegnamento/apprendimento, le SG possono essere considerate delle vere e proprie grammatiche pedagogiche (Andorno, Bosc \& Ribotta, 2003, p. 23), anche se da queste ultime le SG si differenziano per un grado ancora minore di esaustività, dal momento che trattano soltanto le strutture grammaticali - e le relative funzioni - già tematizzate nei capitoli per il lavoro in classe.

Obiettivo del presente contributo è quello di indagare l'apparato concettuale e terminologico impiegato nelle sezioni grammaticali dei manuali di italiano in uso in Germania e la relazione che tale apparato intrattiene con quello della grammatica tradizionale e con i modelli di

1 Alcuni dei manuali che presentano sezioni grammaticali interne al libro dello studente propongono del resto sia una grammatica sistematica in fondo a ciascuno dei volumi di cui si compongono sia delle schede riassuntive a conclusione di ciascun capitolo. 
descrizione della linguistica moderna. Partendo dalla constatazione che la scelta del modello di descrizione grammaticale rappresenta uno dei principali problemi dell'insegnamento linguistico, l'articolo analizzerà un corpus di cinque libri di testo soffermandosi, in particolare, sul trattamento di alcune delle principali categorie morfosintattiche nelle sezioni grammaticali di tali libri. Dopo un paragrafo di inquadramento teorico (2), si forniranno le coordinate metodologiche dell'indagine svolta (3), i cui risultati saranno poi presentati nel paragrafo successivo (4); chiuderà il contributo un bilancio dell'indagine accompagnato da alcune proposte per le sezioni grammaticali dei futuri manuali (5).

\section{QUALE MODELLO DI DESCRIZIONE GRAMMATICALE PER LA CLASSE DI L2/LS?}

Nella progettazione di un sillabo grammaticale per un qualsiasi percorso didattico, sia esso un corso di pochi incontri o un ciclo scolastico di diversi anni, così come nella compilazione di una grammatica o di un manuale di L2/LS, i problemi da affrontare sono molti e di diversa natura. Prima ancora di stabilire quali strutture linguistiche includere nel sillabo è per esempio necessario decidere:

- quale ambito della lingua considerare grammatica; soltanto la morfosintassi, come avviene comunemente, o tutti gli ambiti della lingua in cui siano ravvisabili regolarità, come proposto in ambito italiano da Balboni (2014, pp. 100-101) e da Ciliberti (1991, 2015)?

- di quali varietà sociolinguistiche tenere conto? Solo delle strutture della varietà standard $\mathrm{o}$ anche di quelle ascrivibili a varietà non standard (cfr. p. es. Palermo, 2009, pp. 160-173)?

- a quale apparato concettuale e terminologico fare riferimento? A quello della grammatica tradizionale (GT), presumibilmente più gestibile per docenti e apprendenti ma spesso incoerente al suo interno, non scientificamente fondato e talvolta responsabile di alcune idee distorte sulla lingua diffuse tra parlanti non specialisti/e, o a qualcuno tra i modelli elaborati in seno alla linguistica moderna (LM) come per esempio il modello valenziale, a sostegno del quale - sia per la didattica delle L2/LS che per quella della L1 - si contano numerosi inter- 
venti (p. es. Andorno, 2008, pp. 127-129; Camodeca, 2011; Andorno, 2014 , p. 130)? O forse al modello tradizionale ripulito dai tratti più contestati e opportunamente integrato dagli apporti provenienti dalla $\mathrm{LM}^{2}$ ?

La domanda relativa all'apparato concettuale è quella che in questa sede ci interessa di più. Per darvi una risposta è necessario tenere conto di diverse variabili: oltre a requisiti come l'attendibilità scientifica, la coerenza e la predittività del modello di descrizione (cfr. Andorno, 2008, pp. 126-127), è importante considerare anche variabili esterne come il background metalinguistico di partenza delle/degli apprendenti, e ovviamente anche dello/a stesso/a insegnante, e gli obiettivi didattici del corso. Con "background metalinguistico" è da intendersi il complesso di conoscenze esplicite che entrambi gli attori del processo glottodidattico hanno accumulato studiando a scuola la grammatica della propria L1 e avvicinandosi, in momenti diversi, ad altre lingue e alla riflessione su di esse; tali conoscenze rappresentano un filtro che apprendenti e insegnante inevitabilmente tendono ad applicare nella descrizione di qualsiasi lingua che imparano e insegnano. L'apparato descrittivo e terminologico conosciuto e usato dai/dalle docenti - soprattutto quando i/le docenti sono parlanti nativi/e della lingua che insegnano - può d'altra parte non coincidere con quello imparato e usato dalle/dagli apprendenti, il quale a sua volta può non coincidere con quello impiegato dai libri di testo e dalle grammatiche scolastiche. Per questo, i/le docenti, così come le autrici e gli autori di materiali didattici, non dovrebbero dare per scontata nessuna nozione e nessun termine tecnico impiegato (cfr. Lo Duca, 2006, pp. 184-189 e Rastelli, 2010, p. 152). Quanto alla terminologia, sarebbe inoltre preferibile, come suggerisce Lo Duca (2006, p. 189), evitare doppie o triple denominazioni di categorie come "nome/sostantivo", "pronomi tonici/liberi" o "complemento oggetto/ complemento diretto", optando sempre per i termini più trasparenti, anche se alcuni dei termini della tradizione, pur non essendo traspa-

2 Per approfondimenti sulle nozioni di GT e LM e sui relativi apparati concettuali e terminologici si rimanda a Vanelli, 2010; Graffi, 2012 e 2015; Salvi, 2013; Colombo \& Graffi, 2017 e alla grammatica di Andorno, 2003. 
renti semanticamente, sono più riconoscibili da parte di parlanti non specialisti/e (si veda il caso di "parti del discorso" da un lato e di "classi lessicali" dall'altro).

Anche gli obiettivi del percorso didattico giocano un ruolo importante nella scelta del modello: nel caso tale percorso miri soltanto a portare le/i discenti a realizzare determinati atti comunicativi e consideri quindi la riflessione metalinguistica esclusivamente funzionale a tali atti, potrà essere sufficiente utilizzare un apparato di nozioni già noto, limitando allo stretto necessario l'esplicitazione delle categorie concettuali e il ricorso a termini tecnici. Nel caso però il percorso di insegnamento/apprendimento si proponga invece di potenziare la consapevolezza (meta)linguistica trasversale degli/delle apprendenti - termine con cui traduciamo l'inglese Language awareness (cfr. Gnutzmann, 1997) - e di stimolarli/le a riflettere sui fenomeni linguistici indipendentemente dalle singole lingue in cui essi compaiono, che è del resto obiettivo fondamentale dell'approccio neocomunicativo (Reinfried, 2001) e degli approcci plurali (Candelier et al., 2012), sarà necessario fornire loro un apparato concettuale e terminologico più sofisticato, ossia "un insieme ridotto ma potente di nozioni che consentano di descrivere [le lingue] non tanto nelle loro specificità individuali, ma piuttosto in quanto esempi di lingua umana, cioè nelle caratteristiche di funzionamento di base che si manifestano in quelle come in altre lingue" (Andorno, 2014, p. 130; cfr. anche Brugé, 2000, p. 47).

\section{LA RICERCA}

Partendo dalle considerazioni svolte nei paragrafi precedenti, la ricerca che qui si presenta si proponeva di indagare in che rapporto l'apparato concettuale e terminologico impiegato dalle SG dei manuali di italiano diffusi in Germania si colloca rispetto a quello della GT e ai modelli della LM

Per poter determinare questa relazione, ci si è concentrati, in particolare, sui seguenti aspetti: 
- modalità di trattazione e definizione delle parti del discorso (PdD);

- presenza o assenza di riferimenti espliciti o impliciti della nozione di sintagma;

- modalità di trattazione e definizione delle funzioni sintattiche di soggetto, predicato, oggetto e complementi;

- tipo di terminologia impiegata.

Nella TABELLA n. 1 sono riportati in dettaglio i nomi dei manuali del corpus, le case editrici e le sigle usate nell'articolo. Dal momento che ognuno dei tomi di cui si compongono i cinque manuali contiene una propria $\mathrm{SG}$, le $\mathrm{SG}$ esaminate sono complessivamente quindici.

Tabella 1: I manuali del corpus

\begin{tabular}{|c|c|c|c|c|c|}
\hline & Nomi dei manuali & Volumi & Editori & Pagine relative alle $\mathrm{SG}$ & Sigle \\
\hline 1. & \multirow{3}{*}{ Allegro } & 1 & \multirow{3}{*}{ Klett } & $152-169$ & AL1 \\
\hline 2. & & 2 & & $168-203$ & AL2 \\
\hline 3. & & 3 & & $152-173$ & AL3 \\
\hline 4. & \multirow{3}{*}{ Chiaro! } & A1 & \multirow{3}{*}{ Hueber } & $187-210$ & $\mathrm{CH} 1$ \\
\hline 5. & & $\mathrm{~A} 2$ & & 189-206 & $\mathrm{CH} 2$ \\
\hline 6. & & B1 & & $197-222$ & $\mathrm{CH} 3$ \\
\hline 7. & \multirow{3}{*}{ Con piacere } & A1 & \multirow{3}{*}{ Klett } & $178-195$ & $\mathrm{CP} 1$ \\
\hline 8. & & $\mathrm{~A} 2$ & & 178-197 & $\mathrm{CP} 2$ \\
\hline 9. & & B1 & & $178-193$ & $\mathrm{CP} 3$ \\
\hline 10. & \multirow{3}{*}{ Espresso } & 1 & \multirow{3}{*}{ Hueber } & $161-196$ & ES1 \\
\hline 11. & & 2 & & $198-212$ & ES2 \\
\hline 12. & & 3 & & $191-216$ & ES3 \\
\hline 13. & \multirow{3}{*}{ Insieme } & A1 & \multirow{3}{*}{ Cornelsen } & $151-166$ & IN1 \\
\hline 14. & & $\mathrm{~A} 2$ & & $152-182$ & IN2 \\
\hline 15. & & B1 & & $137-159$ & IN3 \\
\hline
\end{tabular}


I cinque i manuali presentano le seguenti caratteristiche:

- sono stati pubblicati per la prima volta da case editrici tedesche e quindi si rivolgono a un pubblico presumibilmente formato nel sistema educativo tedesco;

- sono stati realizzati da autori e autrici italiani/e con esperienze di insegnamento in ambito germanofono, ma non sempre provenienti da studi di linguistica;

- sono destinati a un pubblico di apprendenti adulte/i e anziane/i che studiano l'italiano in università popolari, Istituti Italiani di Cultura, sedi della Dante Alighieri e scuole private;

- si compongono di tre tomi, ciascuno dei quali dedicato a uno dei primi tre livelli di competenza del Quadro Comune Europeo di Riferimento per le Lingue (QCER);

- contengono SG interne al manuale la cui struttura non si richiama alla partizione del manuale in lezioni, ma che hanno il carattere di grammatica sistematica (cfr. par. 2).

Considerando la funzione di guida che i libri di testo spesso assumono nella classe di L2/LS, ci pare verosimile ipotizzare che anche le SG orientino in modo significativo i momenti dedicati alla riflessione metalinguistica. Analizzare tali sezioni, quindi, può offrire indirettamente uno spaccato sulla realtà dell'insegnamento grammaticale nella classe di lingua e sulle categorie concettuali in esso impiegate.

\section{RISULTATI DELLA RICERCA}

\subsection{Premessa: aspetti generali delle sezioni grammaticali}

Le SG esaminate ${ }^{3}$ sono suddivise in un numero variabile di paragrafi che va da un minimo di otto (AL2, CP3, ES1) a un massimo di sedici (ES1); a loro volta i paragrafi sono suddivisi in sottoparagrafi. Come le normali grammatiche pedagogiche, le SG contengono frequentemente

3 Questi in dettaglio i titoli delle SG: "Systematische Grammatik" ('grammatica sistematica') in AL e CP, "Grammatikübersicht" ('bilancio grammaticale') in CH, "Grammatik" ('grammatica') in ES e IN. 
tabelle finalizzate alla rappresentazione delle forme di diversi paradigmi ed esempi a corredo delle spiegazioni grammaticali. Quasi tutte le SG contengono infine un'appendice costituita da un glossario dei termini metalinguistici, da una lista o tabella di verbi irregolari e, in un caso (IN), anche una lista delle reggenze dei principali verbi.

Nella scelta dei contenuti trattati, le SG, in linea con le grammatiche scolastiche, includono principalmente argomenti morfosintattici, con una netta prevalenza della morfologia - soprattutto verbale - sulla sintassi. I paragrafi di taglio morfologico sono di norma dedicati alle PdD o a classi lessicali trasversali (interrogativi p. es. AL1, p. 160 o ES1, pp. 180-181; numerali p. es. in IN1, p. 165; connettivi testuali in $\mathrm{CH} 3$, pp. 215-217) o anche a sottoclassi delle $\mathrm{PdD}$ (p. es. il pronome relativo in ES3, p. 200); i relativi sottoparagrafi sono dedicati a ulteriori suddivisioni (come per esempio la classificazione dei verbi in coniugazioni a seconda della vocale tematica), a fenomeni relativi alla flessione (come i tempi verbali nei paragrafi dedicati al verbo) o a particolari costruzioni - come il "fare" causativo - in cui la classe lessicale al centro del paragrafo è coinvolta. In un manuale si trova inoltre un paragrafo sulla formazione delle parole (AL2, AL3). I paragrafi di taglio sintattico trattano invece fenomeni come l'ordine dei costituenti in frasi dichiarative e interrogative (p. es. AL2, pp. 165-166 o IN2, pp. 174-176), il comparativo (ES1, p. 173), il periodo ipotetico (CP3, p. 191), il discorso indiretto (CP 3, pp. 191-192) o la consecutio temporum (IN3, p. 154). Non mancano poi paragrafi sul rapporto tra grafematica e fonologia $(\mathrm{CH} 1$, pp. 190-191; IN1, p. 153; ES1, pp. 164-165 con nozioni di prosodia), su modello di quanto fanno le grammatiche scolastiche o anche le grammatiche scientifiche di taglio tradizionale.

Nella sinossi che segue è possibile vedere $i$ temi a cui sono dedicati i paragrafi e le appendici delle SG; i numeri indicano l'ordine in cui tali temi sono presentati: 
Tabella 2: Temi dei paragrafi delle sezioni grammaticali

\begin{tabular}{l|l|l|l|l|l|l|l|l|l|l|l|l|l|l|l}
\hline & \multicolumn{3}{|c|}{ AL } & \multicolumn{3}{c|}{ CH } & \multicolumn{3}{c|}{ CP } & \multicolumn{3}{c|}{ ES } & \multicolumn{3}{c}{ IN } \\
\hline numero di tomo & 1 & 2 & 3 & 1 & 2 & 3 & 1 & 2 & 3 & 1 & 2 & 3 & 1 & 2 & 3 \\
\hline
\end{tabular}

\begin{tabular}{|c|c|c|c|c|c|c|c|c|c|c|c|c|c|c|c|}
\hline Prosodia & 1 & & & & & & 1 & & & & & & & & \\
\hline $\begin{array}{c}\text { Pronuncia e orto- } \\
\text { grafia }\end{array}$ & & & & 1 & & & & & & 1 & & & 1 & 1 & 1 \\
\hline Sostantivi / Nomi & 2 & & & 2 & 4 & 4 & 2 & 1 & 1 & 2 & & 1 & $2^{\mathrm{a}}$ & $2^{\mathrm{b}}$ & 2 \\
\hline $\begin{array}{l}\text { Plurale dei so- } \\
\text { stantivi }\end{array}$ & & & & & & & & & & 3 & & & & & \\
\hline Articoli & 3 & & 1 & 3 & & & 3 & 2 & & 4 & & & & & \\
\hline Possessivi & 4 & & & 6 & & 3 & 4 & & & & 3 & 4 & & & \\
\hline Aggettivi & 5 & 1 & & 4 & 1 & 1 & 5 & 3 & 2 & 5 & 1 & 2 & & 3 & 3 \\
\hline Avverbi & 6 & & & 11 & 2 & & 6 & & & 7 & & 3 & 6 & 7 & 6 \\
\hline Avverbi di tempo & & & & & & & & & & & 9 & & & & \\
\hline Pronomi & & 2 & 2 & & 3 & 2 & 7 & 4 & 3 & & 4 & 5 & 3 & 4 & 4 \\
\hline Pronomi personali & 7 & & & 5 & & & & & & 8 & & & & & \\
\hline Pronomi relativi & & & & & & & & & & & & 6 & & & \\
\hline Interrogativi & 8 & & & 12 & 8 & & 9 & & & 11 & & & & & \\
\hline Verbi & 9 & 5 & 3 & 9 & 7 & 5 & 10 & 6 & 4 & 12 & 6 & 7 & 4 & 5 & 5 \\
\hline Negazione & 10 & & & 10 & & & 11 & & & 13 & & & & & \\
\hline Preposizioni & 11 & 6 & & 14 & 10 & 7 & 13 & 8 & & 14 & & & 5 & 6 & \\
\hline $\begin{array}{l}\text { Numeri e indicato- } \\
\text { ri temporali }\end{array}$ & 12 & & & 15 & 11 & 8 & 14 & 9 & & 16 & & & 8 & 9 & \\
\hline Numerali ordinali & & 7 & & & & & & & & & & & & & \\
\hline Dimostrativi & & 3 & & 7 & 6 & & & & & 9 & & & & & \\
\hline Indefiniti & & 4 & & 8 & 5 & & 8 & 5 & & 10 & 5 & & & & \\
\hline $\begin{array}{c}\text { Formazione delle } \\
\text { parole }\end{array}$ & & 8 & 8 & & & & & & & & & & & & \\
\hline
\end{tabular}




\begin{tabular}{|c|c|c|c|c|c|c|c|c|c|c|c|c|c|c|c|}
\hline & \multicolumn{3}{|c|}{$\mathrm{AL}$} & \multicolumn{3}{|c|}{$\mathrm{CH}$} & \multicolumn{3}{|c|}{$\mathrm{CP}$} & \multicolumn{3}{|c|}{ ES } & \multicolumn{3}{|c|}{ IN } \\
\hline numero di tomo & 1 & 2 & 3 & 1 & 2 & 3 & 1 & 2 & 3 & 1 & 2 & 3 & 1 & 2 & 3 \\
\hline \multicolumn{16}{|c|}{ Temi } \\
\hline Congiunzioni & & & 4 & 13 & 9 & & 12 & 7 & 5 & 15 & 8 & & & & 7 \\
\hline Periodo ipotetico & & & 5 & & & & & & 6 & & 7 & & & & \\
\hline $\begin{array}{l}\text { Concordanza } \\
\text { tempi }\end{array}$ & & & 6 & & & & & & & & & & & & 9 \\
\hline Discorso indiretto & & & 7 & & & & & & 7 & & & & & & \\
\hline Connettivi testuali & & & & & & 6 & & & & & & & & & \\
\hline $\begin{array}{l}\text { Ordine dei costitu- } \\
\text { enti / Tipi di frase }\end{array}$ & & & & & & & & 10 & & & & & 7 & 8 & 8 \\
\hline Nomi dei secoli & & & & & & & & & 8 & & & & & & \\
\hline Comparativo & & & & & & & & & & 16 & 2 & & & & \\
\hline $\begin{array}{l}\text { Tabella verbi } \\
\text { irregolari }\end{array}$ & $\mathrm{X}$ & $\mathrm{x}$ & $\mathrm{X}$ & & & & $\mathrm{X}$ & $\mathrm{x}$ & $\mathrm{x}$ & $\mathrm{x}$ & $\mathrm{x}$ & & $\mathrm{x}$ & $\mathrm{x}$ & $\mathrm{X}$ \\
\hline $\begin{array}{l}\text { Tabella termino- } \\
\text { logia }\end{array}$ & $\mathrm{X}$ & $\mathrm{x}$ & $\mathrm{X}$ & $\mathrm{x}$ & $\mathrm{x}$ & $\mathrm{x}$ & $\mathrm{x}$ & $\mathrm{x}$ & $\mathrm{x}$ & $\mathrm{x}$ & $\mathrm{x}$ & & $\mathrm{x}$ & $\mathrm{x}$ & $\mathrm{X}$ \\
\hline $\begin{array}{c}\text { Tabella reggenze } \\
\text { verbi }\end{array}$ & & & & & & & & & & & & & & $\mathrm{x}$ & $\mathrm{X}$ \\
\hline
\end{tabular}

${ }^{a}$ Il paragrafo è dedicato in realtà non solo al nome, bensì al nome e agli elementi che lo accompagnano.

${ }^{\mathrm{b}}$ Come sopra.

Le SG di tutti i manuali sono scritte interamente in tedesco; l'italiano è usato soltanto negli esempi, accompagnati in alcuni manuali dalla traduzione tedesca, e nei nomi di singole strutture linguistiche. Per quanto riguarda le lingue tematizzate, le SG sono, come del resto i manuali che le ospitano, tendenzialmente monolingui, nel senso che illustrano quasi sempre i fenomeni linguistici dell'italiano senza fare riferimento ad altre lingue, se non, occasionalmente, al tedesco (cfr. p. es. AL2, p. 154, in cui si sottolinea come la serie di pronomi tonici e atoni dell'italiano rappresenti una differenza rispetto al tedesco; oppure $\mathrm{CP} 2$, 
p. 196, in cui si spiegano le nozioni di oggetto diretto e indiretto facendo riferimento ai casi del tedesco accusativo e dativo).

4.2. Modalità di trattazione delle parti del discorso

\subsubsection{Distribuzione nei volumi dei manuali}

Come si può vedere dalla tabella n. 2, non in tutti i tomi di cui si compongono i manuali si hanno paragrafi per ciascuna delle PdD e questo perché, come già detto, le SG contengono solo gli argomenti grammaticali esplicitamente tematizzati nei capitoli per il lavoro in classe. Le uniche PdD trattate sistematicamente in tutte le SG del corpus sono il verbo $(\mathrm{V})$ e il pronome $(\mathrm{Pro})^{4}-$ le due classi più complesse dal punto di vista flessionale - seguite dall'aggettivo (Agg) (presente in tredici SG su quindici), dal nome (N) (presente in dodici SG), dalla preposizione (Prep) e dall'avverbio (Avv) (dieci presenze), dalla congiunzione (Cong) (nove presenze) e dall'articolo (Art) (sei presenze).

Notiamo en passant che in nessuno dei manuali del corpus sono presenti paragrafi sull'interiezione, scelta che ci pare sintomatica di una scarsa attenzione riservata dai manuali al parlato.

\subsubsection{Classificazione delle parti del discorso e modalità di presentazione di alcune classi trasversali}

Il fatto che alcune SG scelgano di dedicare paragrafi autonomi a classi trasversali come possessivi (Poss), dimostrativi (Dim) o indefiniti (Indef), che possono avere funzione sia di Pron che di determinanti/specificatori (cfr. Colombo \& Graffi, 2017, pp. 130-136 e Andorno, 2003, pp. 19-28) o, nella prospettiva della GT, di aggettivi, così come a sottoclassi di singole PdD (come ad esempio i Pron relativi in $\mathrm{CH} 3$ ), non deve indurre a pensare che in tali sezioni queste classi siano automaticamente considerate $\mathrm{PdD}$ a sé stanti. Si dovrà piuttosto ipotizzare che

4 Nel computo si sono accorpati i dati relativi ai paragrafi genericamente dedicati al Pro, quelli dedicati ai soli Pro personali e quelli dedicati ai soli relativi.

5 Nel calcolo si sono accorpati i dati relativi ai paragrafi genericamente dedicati all'Avv e al paragrafo della SG di ES2 dedicato agli Avv di tempo. 
la trattazione autonoma sia finalizzata a facilitare la consultazione delle SG. Detto questo, pare tuttavia interessante osservare come le modalità di trattazione di classi come Dim, Poss, Indef varino da manuale a manuale: AL, CH e ES scelgono per esempio di trattare Art, Poss, Dim e Indef in quattro paragrafi separati; CP sceglie invece di trattare separatamente Art, Poss e Indef e di inserire i Dim, solo nel loro uso pronominale, all'interno del paragrafo dedicato ai Pro; IN opta infine per una soluzione più articolata, ma forse meno coerente: IN1 propone un paragrafo intitolato Das Nomen und seine Begleiter (Il nome e gli elementi che lo accompagnano) in cui colloca N, Art, Poss e Agg (solo qualificativo) e dedica un paragrafo separato al Pro; IN2 propone anch'esso un paragrafo sul nome e sugli elementi che lo accompagnano, inserendovi però solo N, Art e Poss, mentre dedica un paragrafo autonomo all'Agg con sottoparagrafi dedicati a Dim e Indef. Il paragrafo cumulativo scompare invece in INS3.

\subsubsection{Definizioni delle parti del discorso}

A differenza di quanto accade nelle grammatiche descrittive e nelle grammatiche pedagogiche per nativi/e, in cui ogni capitolo o paragrafo dedicato alle $\mathrm{PdD}$ è aperto da una definizione, i corrispondenti paragrafi delle SG sono caratterizzati dalla presenza non uniforme di definizioni: se categorie come quelle degli Avv e Agg tendono a essere quasi sempre definite, anche perché di difficile individuazione per apprendenti germanofone/i, di $\mathrm{Art}^{6} \mathrm{e} \mathrm{V}$ non viene mai fornita una definizione, mentre dei $\mathrm{N}$ si trova solo una definizione. Coerentemente con gli obiettivi didattici delle SG, inoltre, le definizioni presenti - sia quelle delle PdD che quelle delle relative sottoclassi (Pron personali, relativi, avverbiali ecc.) - tendono a non essere esaustive: dei Dim, per esempio, è sempre evidenziata la funzione deittica, quasi mai quella anaforico-testuale; dei Pron personali è trattata sempre la funzione anaforica, mai quella cataforica. Questa non esaustività è giustificata dal fatto che i manuali in cui sono comprese le SG esaminate coprono solo i primi tre livelli di

6 I paragrafi dedicati all'Art sono però aperti, in alcuni manali (p.e. CH1, p. 193), da indicazioni relative all'accordo con il $\mathrm{N}$. 
competenza del QCER e che quindi spesso non trattano funzioni meno prototipiche, considerate meno urgenti da un punto di vista comunicativo. La non esaustività delle definizioni può tuttavia ridursi man mano che si sale di livello: le definizioni presenti nelle SG dei volumi destinati ai livelli A2 e B1 tendono per esempio a essere più complesse di quelle presenti nelle SG dei volumi A1. C'è comunque da dire che in alcuni casi la non esaustività delle definizioni può tradursi in una eccessiva genericità: ne è un esempio la definizione delle Prep proposta da $\mathrm{CH} 1$ (206), secondo cui queste ultime "collegano diversi elementi di una frase", che potrebbe essere applicata anche alle Cong.

Nelle definizioni delle PdD le SG non adottano criteri uniformi: talvolta ricorrono, come la GT, a criteri semantico-nozionali ("Gli Agg sono parole esprimenti qualità, che caratterizzano più precisamente una persona o una cosa", CP1, p. 183), talvolta a criteri sintattici ("I Pron personali vengono usati al posto di $\mathrm{N}$ comuni e $\mathrm{N}$ propri e possono avere la funzione di soggetto e oggetto", IN1, p. 157), talaltra a criteri misti, cioè combinano criteri semantici con criteri sintattici ("I $\mathrm{N}$ indicano cose o persone e vengono quasi sempre accompagnati da altre parole", IN1, p. 153). La classe degli Indef è definita da due manuali (AL e CP) attraverso l'elencazione di alcune delle sue sottoclassi. Un manuale (AL) sceglie occasionalmente di inserire nelle definizioni il termine scolastico tedesco relativo alla parte del discorso definita (p. es. "I Pron personali sono persönliche Fürwörter che possono assumere la posizione di un soggetto o oggetto", AL1, p. 159)

Nella TABELLA n. 3 vengono riassunti i criteri adottati per ciascuna PdD, includendo anche le classi trasversali trattate in paragrafi autonomi:

7 Tutte le traduzioni dal tedesco sono mie. 
Tabella 3: Criteri adottati nelle definizioni delle PdD

\begin{tabular}{|c|c|c|c|c|c|c|c|c|c|c|c|c|}
\hline Manuale & Vol & $\mathrm{N}$ & V & Pro & Art & Agg & Avv & Prep & Cong & Dim & Indef & Poss \\
\hline \multirow{3}{*}{$\mathbf{A L}$} & 1 & & & CSD & & & $\mathrm{CSN}$ & & & & & \\
\hline & 2 & & & CSD & & & & & & CSD & $\mathrm{SC}$ & \\
\hline & 3 & & & & & & & & CSD & & & \\
\hline \multirow{3}{*}{ CH } & 1 & & & & & $\mathrm{CSN}$ & $\mathrm{CSN}$ & CSD & CSD & & & \\
\hline & 2 & & & & & $\begin{array}{l}\text { CSN } \\
\text { CSD }\end{array}$ & $\mathrm{CSN}$ & CSD & & & $\mathrm{CSN}$ & \\
\hline & 3 & & & $\mathrm{CSD}^{\mathrm{a}}$ & & & & & & & & \\
\hline \multirow{3}{*}{$\mathrm{CP}$} & 1 & $\mathrm{CM} ?$ & & CSD & & $\mathrm{CSN}$ & $\mathrm{CSN}$ & & CSD & & $\mathrm{SC}$ & \\
\hline & 2 & & & $\begin{array}{c}\text { CSD } \\
\text { CSD }^{\mathrm{b}}\end{array}$ & & & & & & CSD & $\mathrm{SC}$ & \\
\hline & 3 & & & & & & & & CSD? & & & \\
\hline \multirow{3}{*}{ ES } & 1 & & & & & & $\mathrm{CSN}$ & CSD & CSD & & & \\
\hline & 2 & & & & & & & & & & $\begin{array}{c}\text { CSN } \\
\text { CSD? }\end{array}$ & \\
\hline & 3 & & & & & & & & & & & \\
\hline \multirow{3}{*}{ IN } & 1 & $\begin{array}{l}\mathrm{CSN} / \\
\mathrm{CSD}\end{array}$ & & $\mathrm{CSN} / \mathrm{CSD}$ & & $\mathrm{CSN}$ & $\begin{array}{l}\text { CSD } \\
\text { CM }\end{array}$ & & & & & $\mathrm{CSN}$ \\
\hline & 2 & & & & & $\mathrm{CSN}$ & & & & & & \\
\hline & 3 & & & & & $\mathrm{CSN}$ & & & $\mathrm{CSD}^{\mathrm{c}}$ & & & \\
\hline
\end{tabular}

Legenda: $\mathrm{CSN}=$ criteri semantico-nozionali; $\mathrm{CM}=$ criteri morfologici; $\mathrm{CSD}=$ criteri sintattico-distribuzionali; $\mathrm{SC}=$ elencazione di sottoclassi

${ }^{a}$ La definizione si riferisce alla sola categoria dei Pro relativi.

${ }^{\mathrm{b}} \mathrm{Nel}$ paragrafo vengono riportate due definizioni, relative alle sottoclassi dei Pron personali e relativi.

${ }^{\mathrm{c}}$ La definizione, che non ha la Cong come soggetto, è così formulata: "Frasi e porzioni di frasi vengono collegate (tra loro) grazie alle Cong" (IN3, p. 150).

Si può dire, nel complesso, che i criteri semantico-nozionali tendono a prevalere con le classi lessicali semanticamente piene e quelli 
sintattico-distribuzionali con gli elementi funzionali. Rare, come si può vedere, sono le definizioni che fanno riferimento a criteri morfologici.

\subsection{Presenza o assenza di riferimenti alla nozione di sintagma}

La nozione di sintagma, ignorata dalle grammatiche scolastiche e spesso conosciuta solo in modo intuitivo da docenti e apprendenti, non è tematizzata in nessuna SG. Qualche riferimento implicito si trova soltanto in alcuni paragrafi dedicati alle Cong e alle Prep, laddove si spiega che queste PdD servono a collegare Satzglieder (costituenti di frase) (cfr. $\mathrm{CH} 1$, p. 206; CH2, p. 204) o nei paragrafi sull'ordine dei costituenti, in cui si parla genericamente di Satzteile (parti di frase) (IN1, p. 163), oltre che nel citato paragrafo sul $\mathrm{N}$ e sugli elementi che lo accompagnano in INS1. Nelle definizioni relative ai Pron personali, in vari manuali si fa riferimento al fatto che questi possano sostituire un soggetto o un oggetto diretto o indiretto e quindi un gruppo di parole. Di quali siano i criteri per stabilire perché tra alcune parole, nella frase, sussista un rapporto più stretto rispetto a quello che sussiste tra altre parole e di quali siano le differenti proprietà di questi gruppi di parole a seconda della PdD che ne è la testa, non si occupa però nessun manuale.

\subsection{Classificazione e definizione delle funzioni sintattiche}

Nessuna delle SG dedica paragrafi separati alle funzioni sintattiche dei costituenti, la cui conoscenza è data per scontata sulla base della competenza metalinguistica di partenza. Tutti i manuali però fanno riferimento alle funzioni di soggetto ( $\mathrm{Sog}$ ) e di oggetto $(\mathrm{Ogg})$ (diretto e indiretto), come appena detto, nei paragrafi dedicati ai Pro personali e relativi (cfr. p. es. AL2, p. 156; CP1, p. 185; IN2, p. 152) e nelle appendici dedicate alle reggenze dei V: nei paragrafi sui Pro personali si sottolinea come l'espressione del Pro Sog in italiano non sia obbligatoria (cfr. p. es. IN1, p. 157) e come i Pro con funzione di Ogg diretto e indiretto possano essere sia tonici che atoni (cfr. p. es. AL2, p. 154). Nei paragrafi sui relativi si sottolinea invece che il Pro "che" può avere sia la funzione di Sog che quella di Ogg, senza però esplicitare anche qui i criteri per il loro riconoscimento nella frase; nelle liste di IN sulle reggenze 
dei V, gli indefiniti "qualcosa", "qualcuno" e "a qualcuno" si riferiscono implicitamente alle funzioni di Ogg diretto e indiretto. Quattro manuali (AL3, p. 182; CH3, p. 210; ES3, p. 211; IN3, p. 150) su cinque scelgono infine di non fare riferimento alle funzioni di Sog e Ogg nei paragrafi dedicati alla costruzione passiva, ma di menzionare esplicitamente solo il ruolo tematico dell'agente (in tedesco Urheber nei primi quattro manuali o Handlungsurheber in IN3): i manuali spiegano infatti che, nella costruzione passiva, l'agente dell'azione (Urheber der Handlung) è preceduto dalla Prep "da".

Per quanto riguarda infine l'aspetto più tipico della GT, soprattutto della sua variante italiana, ossia il "folkloristico" inventario di complementi, ripetutamente criticato (cfr. tra i tanti Simone \& Cardona, 1971, pp. 383-386; Graffi, 2012, pp. 79-89) se non addirittura fatto oggetto di scherno (cfr. p. es. Colombo, 1997) nell'ambito della LM, ne troviamo tracce solo indirette: nei paragrafi sulle Prep è presente, per esempio, una lista con le principali funzioni di ciascuna Prep, che sembra ricalcare i classici schemi dei complementi. Si veda per esempio la figura che segue, tratta da IN2 (p. 173):

\subsection{Terminologia metalinguistica}

Le scelte terminologiche compiute dalle SG sono caratterizzate da un certo eclettismo. Dal metalinguaggio internazionale di origine latina provengono i nomi delle PdD (Substantiv - sostantivo; Verb - verbo; Adverb - avverbio etc.), delle funzioni sintattiche (Subjekt- soggetto; Objekt - oggetto ecc.), dei casi tedeschi (Nominativ - nominativo; Genitiv - genitivo ecc.) e di nozioni sintattiche come reflexiv (riflessivo) o Passiv (passivo). Occasionalmente sono presenti però anche termini della tradizione scolastica tedesca, consistenti interamente di materiale lessicale germanico: è il caso, per esempio, di nomi di PdD come Hauptwort (parola principale) per 'nome', Eigenschaftswort (parola indicante qualità) per 'aggettivo', Fürwort (proparola) per 'pronome' o di altri termini come rückbezüglich per 'riflessivo', Selbstlaut (suono autonomo) per 'vocale' o Mitlaut (consuono) per 'consonante'; tali nomi ricorrono soprattutto nelle definizioni, come già sottolineato, e nei glossari metalinguistici, nei quali i termini di origine latina vengono spesso tra- 
Figura 1: Sottoparagrafo sulle Prep semplici in IN2

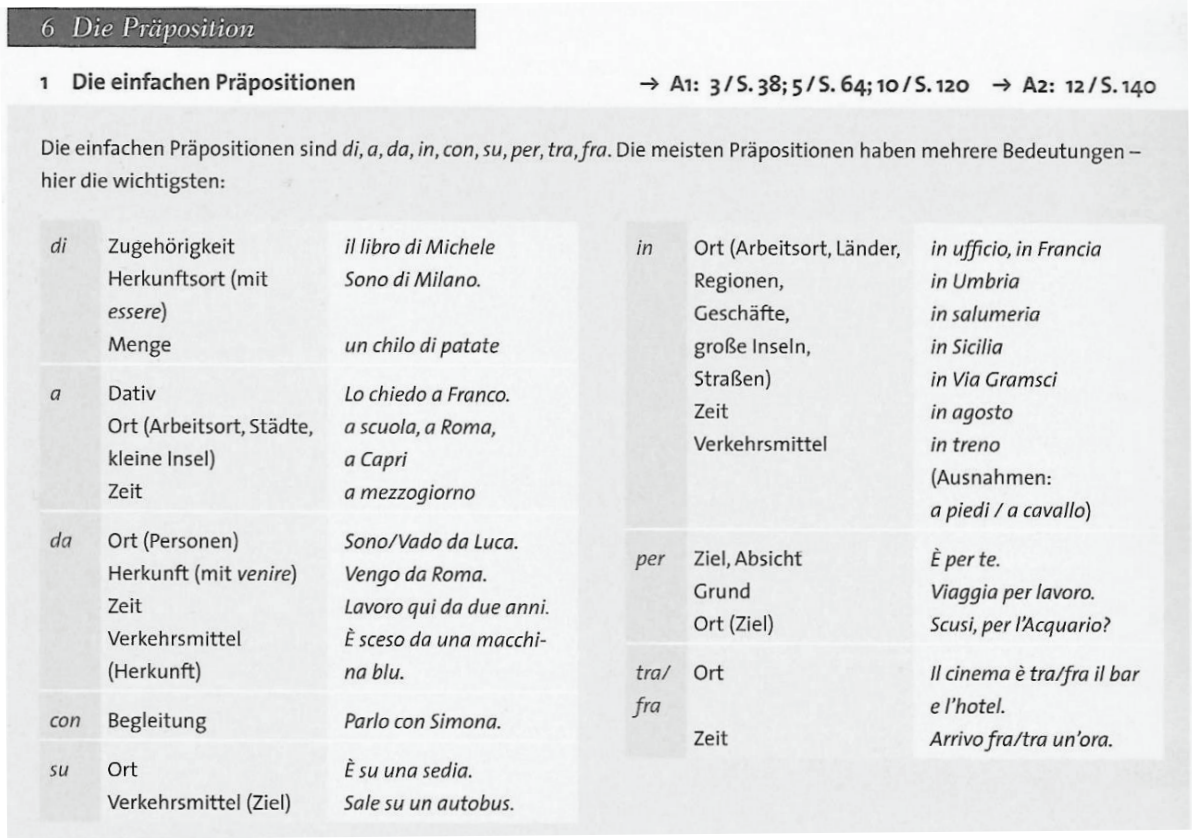

dotti con quelli germanici. Alla GT italiana sono riconducibili invece i nomi dei tempi e dei modi del V ("passato prossimo", "imperfetto", "congiuntivo", "condizionale passato", ecc.), quasi sempre riportati in italiano, alcuni calchi come absoluter e relativer Superlativ (superlativo assoluto e relativo) e i nomi "complemento oggetto" e "complemento di termine", presentati nei soli glossari dei manuali AL e CP (cfr. AL2, p. 172; CP3, p. 192) rispettivamente come equivalenti di direktes Objekt (oggetto diretto) o Akkusativ (accusativo) e di indirektes Objekt (oggetto indiretto) o Dativ (dativo).

Nei casi di divergenza tra la terminologia della LM e quella della GT - si pensi per esempio ai nomi dei tempi verbali del passato - i manuali preferiscono optare per quest'ultima. 


\section{OSSERVAZIONI CONCLUSIVE}

Le SG prese in esame hanno come utenti di riferimento un pubblico genericamente adulto, iscritto ai corsi delle università popolari o di altre istituzioni formative dell'Erwachsenenbildung tedesca. Questo pubblico, nonostante quello che si potrebbe pensare esaminando i manuali, non rappresenta una realtà omogenea, potendo comprendere profili di apprendenti assai diversi per età, livello culturale, classe socio-economica, pregresse esperienze di apprendimento linguistico, motivazioni per lo studio dell'italiano, obiettivi di apprendimento. D'altro canto, anche i/le docenti che usano questi manuali rappresentano un gruppo assai composito, che può comprendere sia nativi/e che non nativi/e, provenienti dai più diversi percorsi universitari e dalle più disparate esperienze lavorative. Se pensiamo all'apparato concettuale e terminologico impiegato nelle SG analizzate e al possibile impiego concreto di tali sezioni, ci sembra che esso tenga conto solo di una parte delle/dei potenziali utenti dei manuali: quella cioè costituita da insegnanti e apprendenti con un bagaglio metalinguistico (presumibilmente) coincidente con quello della GT appresa a scuola e che non considerano un obiettivo primario del corso di lingua quello di mettere in discussione o ampliare tale bagaglio di nozioni; per queste/i insegnanti e apprendenti le categorie concettuali presenti nelle SG rappresentano uno strumento pratico da innestare sul bagaglio metalinguistico di partenza in funzione del raggiungimento di non sempre ben precisati obiettivi comunicativi. Dal momento che l'apparato concettuale delle $\mathrm{SG}$ - così come è stato descritto nei paragrafi precedenti - rappresenta una versione ripulita di quello della GT, esso può essere gestibile sia da apprendenti formati/e nel sistema educativo tedesco sia da docenti germanofoni/e e italofoni/e con i requisiti appena visti. D'altra parte, lo stesso apparato può rivelarsi a nostro avviso inadeguato - o, comunque, bisognoso di massicce integrazioni - per due gruppi di insegnanti e discenti: in primo luogo, per quelli/e che dispongono di un bagaglio metalinguistico ancora più limitato di quello rappresentato dalla GT e che avrebbero per questo bisogno di definizioni più esplicite delle nozioni impiegate così come di indicazioni 
più chiare per quanto riguarda i criteri adottati per l'identificazione delle PdD e delle funzioni sintattiche e, di conseguenza, la terminologia usata. In secondo luogo, le categorie usate nelle SG possono rivelarsi altrettanto inadeguate per insegnanti e apprendenti interessati/e a potenziare la propria capacità di osservazione dei (e di riflessione sui) fenomeni linguistici, anche al di là della contingenza della lingua studiata, superando contemporaneamente i modelli di descrizione imparati a scuola. Per venire incontro ai bisogni di un siffatto pubblico, nelle SG di futuri manuali sarebbe a nostro avviso opportuno:

- definire in modo più esplicito e attraverso criteri scientificamente fondati le nozioni concettuali a cui si fa ricorso, anche mettendo in discussione - $\mathrm{o}$, al contrario, anche riconoscendone la parziale utilità pedagogica - le definizioni della GT ed eventualmente confrontando le definizioni e la terminologia impiegate nelle tradizioni scolastiche italiane e tedesche;

- fornire strumenti di analisi dei fenomeni grammaticali diversi da quelli tradizionali, introducendo la nozione di sintagma e gli elementi di base di modelli come quello della grammatica valenziale, la cui utilità in sede didattica è stata sottolineata da più parti;

- rendere oggetto di focalizzazione i cosiddetti fenomeni di interfaccia, come per esempio le dislocazioni, esplicitando i rapporti che intercorrono tra funzioni sintattiche, ruoli tematici e struttura informativa dell'enunciato;

- allargare il concetto di grammatica a tutti gli ambiti della lingua, anche esterni alla morfosintassi, in cui siano ravvisabili regolarità si pensi, per esempio, agli ambiti testuale e conversazionale - così come a registri diversi dalla varietà standard; tentare, insomma, di far capire che la grammatica è soprattutto la descrizione dei comportamenti sistematici dei/delle parlanti piuttosto che una sorta di codice penale del linguaggio;

- stimolare a riflettere le/gli utenti delle SG sull'applicabilità delle categorie di descrizione impiegate per l'italiano nella descrizione di altre lingue, superando la concezione monolingue che caratterizza le attuali SG; 
- fare sistematico riferimento, coerentemente con i principi degli approcci plurali, ad altre lingue (tedesco, lingue romanze, inglese, latino, ma anche lingue geneticamente o tipologicamente più distanti presenti nello spazio linguistico tedesco che possono offrire interessanti basi di transfer) per spiegare le regole dell'italiano, evidenziandone somiglianze, differenze e - limitatamente al latino - eventuali rapporti di derivazione.

\section{BIBLIOGRAFIA}

Andorno, C. (2003). La grammatica italiana. Milano: Bruno Mondadori.

Andorno, C. (2008). Insegnare e imparare l'italiano L2: quale grammatica? In R. Grassi, R. Bozzone Costa \& C. Ghezzi (eds.), Dagli studi sulle sequenze di acquisizione alla classe di italiano L2 (pp. 123-142). Perugia: Guerra.

Andorno, C. (2014). Grammatica e competenza metalinguistica. In M. Chini $\&$ C. Bosisio (eds.), Fondamenti di glottodidattica. Apprendere e insegnare le lingue oggi (pp. 115-131). Roma: Carocci.

Andorno, C., Bosc, F. \& Ribotta, P. (2003). Grammatica. Insegnarla e impararla. Perugia: Guerra.

Balboni, P.E. (2014). Didattica dell'italiano come lingua seconda e straniera. Torino: Bonacci-Loescher.

Brugé, L. (2000). Teoria linguistica e insegnamento della grammatica. In R. Dolci \& P. Celentin (eds.). La formazione di base del docente di italiano a stranieri (p. 42-61). Roma: Bonacci.

Camodeca, C. (2011). La grammatica valenziale nella classe di italiano L2. Una sperimentazione. In L. Corrà \& W. Paschetto (eds.). Grammatica a scuola (pp. 273-283). Milano: Franco Angeli.

Candelier, M., Camilleri-Grima, A., Castellotti, V., de Pietro, J.-F., Lörincz, I., Meißner, F.-J., Schröder-Sura, A., Noguerol, A. \& Molinié, M. (2012). Le CARAP - Un Cadre de référence pour les approches plurielles, Compétences et ressources. Graz: Council of Europe Publishing/Centre européen pour l'enseignement des langues. Retrieved from goo.gl/odxCBN.

Ciliberti, A. (1991). Grammatica, pedagogia, discorso. Firenze: La Nuova Italia.

Ciliberti, A. (2015). La grammatica: modelli per l'insegnamento. Roma: Carocci. 
Colombo, A. (1997). La riflessione sulla lingua dalla scuola elementare al biennio superiore, La didattica, 4, 77-82.

Colombo, A. \& Graffi, G. (2017). Capire la grammatica. Il contributo della linguistica. Roma: Carocci.

Gnutzmann, C. (1997). Language Awareness. Geschichte, Grundlagen, Anwendungen. Praxis des neusprachlichen Unterrichts, 44(3), 227-236.

Graffi, G. (2012). La frase: l'analisi logica. Roma: Carocci.

Graffi, G. (2015). Teorie linguistiche e insegnamento della grammatica. In M.E. Favilla \& E. Nuzzo (eds.), Grammatica applicata: apprendimento, patologie, insegnamento (pp. 197-212). Bologna: AITLA. Retrieved from goo.gl/cdMaEY.

Lo Duca, M.G. (2006). Quale lessico per facilitare la riflessione formale sull'italiano L2? In F. Caon (ed), Facilitare l'apprendimento dell'italiano L2 e delle lingue straniere (pp. 180-191). Torino: Utet.

Palermo, M. (2009). Quale italiano? In P. Diadori, M. Palermo \& D. Troncarelli, Manuale di didattica dell 'italiano L2 (pp. 160-173). Perugia: Guerra.

Rastelli, S. (2010). La didattica acquisizionale: ragioni, metodo e critica. In S. Rastelli (ed.), Italiano di cinesi, italiano per cinesi dalla prospettiva della didattica acquisizionale. Perugia: Guerra.

Reinfried, M. (2001). Neokommunikativer Fremdsprachenunterricht: ein neues methodisches Paradigma. In F.J. Meißner \& M. Reinfried (eds.), Bausteine für einen neokommunikativen Französischunterricht. Lernerzentrierung, Ganzheitlichkeit, Handlungs-orientierung, Interkulturalität, Mehrsprachigkeitsdidaktik (pp. 1-20). Tübingen: Narr.

Salvi, G. (2013). Le parti del discorso. Roma: Carocci.

Simone, R. \& Cardona, G.R. (1971). Strutture teoriche di alcune grammatiche scolastiche italiane. In M. Medici \& R. Simone (eds.), L'insegnamento dell'italiano in Italia e all'estero (pp. 365-393). Bulzoni: Roma.

Vanelli, L. (2010). Grammatiche dell'italiano e linguistica moderna. Padova: Unipress.

\section{Corpus}

AL1: Merklinghaus, R. et al. (2003). Allegro 1. Lehr- und Arbeitsbuch. Stuttgart: Klett.

AL2: Merklinghaus, R. et al. (2003). Allegro 2. Lehr- und Arbeitsbuch. Stuttgart: Klett.

AL3: Merklinghaus, R. et al. (2005). Allegro 3. Lehr- und Arbeitsbuch. Stuttgart: Klett. 
CH1: De Savorgnani G. \& Bergero, B. (2009). Chiaro! A1. Der Italienischkurs. Ismaning: Hueber.

CH2: De Savorgnani, G. \& Codera Alberti, C. (2011). Chiaro! A2. Der Italienischkurs. Ismaning: Hueber.

CH3: De Savorgnani, G. \& Codera Alberti, C. (2012). Chiaro! B1. Der Italienischkurs. Ismaning: Hueber.

CP1: Zorzan, L. et al. (2010). Con piacere A1. Lehr- und Arbeitsbuch Italienisch. Stuttgart: Klett.

CP2: Zorzan, L. et al. (2011). Con piacere A2. Lehr- und Arbeitsbuch Italienisch. Stuttgart: Klett.

CP3: Finzi, A. et al. (2013). Con piacere B1. Lehr- und Arbeitsbuch Italienisch. Stuttgart: Klett.

ES1: Ziglio, L. \& Rizzo, G. (2009). Espresso 1. Lehr- und Arbeitsbuch. Erweiterte Ausgabe. Insmaning: Hueber.

ES2: Balì, M. \& Rizzo, G. (2009). Espresso 2. Lehr- und Arbeitsbuch. Erweiterte Ausgabe. Ismaning: Hueber.

ES3: Balì, M. \& Ziglio, L. (2010). Espresso 3. Lehr- und Arbeitsbuch. Erweiterte Ausgabe. Ismaning: Hueber.

IN1: Colombo, F. et al. (2008). Insieme A1. Kurs- und Arbeitsbuch. Berlin: Cornelsen.

IN2: Colombo, F. et al. (2009). Insieme A2. Kurs- und Arbeitsbuch. Berlin. Cornelsen: 2009.

IN3: Faraci, C. et al.(2010). Insieme B1. Kurs- und Arbeitsbuch. Berlin: Cornelsen.

Riassunto: Il presente contributo analizza l'apparato concettuale e terminologico impiegato nelle sezioni grammaticali dei manuali di italiano per apprendenti adulti/e di madrelingua tedesca. L'articolo si propone in particolare di stabilire che tipo di relazione queste sezioni intrattengono con l'apparato concettuale della grammatica tradizionale (GT) e con i modelli della linguistica moderna (LM). L'analisi considera in particolare le seguenti variabili: 1) trattamento delle parti del discorso con particolare attenzione per la modalità di presentazione e per i criteri di definizione; 2) presenza o assenza di riferimenti espliciti o impliciti alla nozione di sintagma; 3) trattamento delle funzioni sintattiche di soggetto, oggetto diretto e indiretto e complementi; 4) terminologia impiegata. Dall'analisi scaturiscono le seguenti conclusioni: le differenze più vistose nel modo in cui vengono trattate le parti del discorso riguardano gli articoli e le classi trasversali come dimostrativi, indefiniti e possessivi; le definizioni delle parti del discorso non seguono criteri omogenei; i riferimenti al concetto di sintagma-fondamentale nella LM e sconosciuta o ignorata nella GT-sono solo impliciti; le funzioni di soggetto, oggetto e complementi sono più volte citate senza che, però ne vengano fornite definizioni esplicite; le scelte terminologiche presentano infine un certo grado di eclettismo, 
dal momento che includono sia termini della terminologia metalinguistica internazionale sia termini della tradizione scolastica tedesca e italiana.

Parole chiave: grammatica, manuali di italiano, parti del discorso, funzioni sintattiche, terminologia metalinguistica 